\title{
Depth of Invasion as a Potential Predictor of Recurrence in Patients Treated with Enbloc -Transurethral Resection of the Bladder: A Feasibility Study
}

Roberto Giulianelli*1, Ludovica De Vincentis*1, Barbara Cristina Gentile ${ }^{1}$, Stefania Monclesi ${ }^{1}$, Gabriella Mirabile ${ }^{1}$, Luca Albanesi ${ }^{1}$, Paola Tariciotti ${ }^{1}$, Giorgia Tema ${ }^{1}$, Giorgio Rizzo ${ }^{1}$, Antonio Nacchia ${ }^{1}$, Pietro Aloisi ${ }^{1}$, Giorgio Vincenti $^{1}$, Riccardo Lombardo ${ }^{2}$ and Giuseppe Soda ${ }^{1}$

${ }^{1}$ Department of Pathology, Italy

${ }^{2}$ Department of Urology, Italy

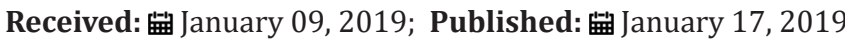

*Corresponding author: Roberto Giulianelli, Department of Pathology, Italy

Ludovica De Vincentis, Department of Pathology, Italy

Abstract

Background: As for other tumors it is likely that depth of invasion is a prognostic factor for disease recurrence and progression in high grade pT1 urothelial bladder cancer. To date nor anatomy based neither dimensional subclassification proved reliable correlation with recurrence and progression, mainly considering the high interobserver variability in pT1 diagnosis, based by the TURB resection technique intrinsic artifact.

Objective: Aim of this study is to assess the feasibility of measuring depth of invasion of urothelial carcinoma in patients undergoing EB-TURB for $\mathrm{pT} 1 \mathrm{HG}$ disease.

Design, Setting, and Participants: 27 patients undergoing EB-TURB with Collins knife and with pT1-HG disease were included. A second TURB was performed after 4-6 weeks from the first one. A dedicated pathologist assesses the feasibility of depth of invasion measurement.

Outcome Measurements and Statistical Analysis: Evaluate depth of invasion using Kruskal Wallis analysis and Chi-square test.

Results and limitations: Overall 32 patients with pT1HG disease were enrolled. EB-resection was adequately performed in $27 / 32$ (85\%) of the patients. Overall 40 lesions were identified with a median tumour size of $2 \mathrm{~cm}(1 / 4)$. Median depth of invasion was $1.35 \mathrm{~mm}(0.48 / 3.5)$. Deepness measurement was feasible in $100 \%$ of the patients and in $100 \%$ of the lesions. As well on re TURB $8 / 27(29 \%)$ patients presented residual disease and in $100 \%$ of these patients it was possible to measure depth of invasion with a median value was $1.1 \mathrm{~mm}(0.43 / 2.3)$. Limitations include number of patients.

Conclusion: In specimen obtained from EB-TURB measurement assessment proved to be easy and highly reproducible. Recruitment of patients is still ongoing to evaluate an eventual prognostic value of neoplastic invasion in recurrence and progression.

Patient Summary: In this report we want to find out if the thickness of invasion of urothelial carcinoma is related to tumor recurrence and progression measuring depth of invasion.

Keywords: Enbloc-Transurethral Resection of the Bladder; Depth of Invasion; Recurrence 


\section{Introduction}

Bladder urothelial carcinoma is the fourth most common cancer in men and the tenth in women [1]. Despite the knowledge of its potential risk, T1 non muscle invasive bladder carcinoma shows a significant variability from patient to patient in term of disease recurrence and progression and consequently in clinical and surgical approach [2]. High grade NMIBC patient's treatment is the most controversial, especially regarding surgical timing: authors recommend radical cystectomy for all T1, encouraged by the significant risk of progression and the disease specific mortality, thus reserving an overtreatment for non-progressive patients [3], whereas others support the role of adjuvant BCG therapy, with subsequent cystectomy just in case of recurrence [2]. To enlighten which patient should benefit one treatment upon another, increasing interest has been given in study of outcome predictive variable in term of risk of recurrence and progression. EORTC risk table are the best-established models to predict recurrence and progression in NMIBC patients: six clinico-pathological variable including multiplicity, tumor size, T category, tumor grade, prior recurrence rate and presence of concurrent CIS are the most suitable to predict tumor behavior [4]. All these parameters are useful but actually not fully satisfactory in discriminating disease recurrence and progression [5].

Lively debate and active research on this topic moved the focus to lamina propria invasion, investigating the role of muscularis mucosae: stratification by muscularis mucosae invasion is the most studied sub-staging systems and its prognostic value was confirmed by many studies, and even if none have been whole satisfactory, lamina propria invasion is now accepted as a significant predictor of progression in patients with primary pT1 tumour of the bladder, with a $21.9 \%$ progression rate for $\mathrm{T} 1$ tumours deeply invading the lamina propria [6]. Anyway none of the sub-staging system probed in the past decade has been validated, but despite it is not included in the VII edition of the TNM, it is now strongly recommended by the latest Cancer Staging manual of the American Joint Committee on Cancer to report an attempt to categorize pT1 diseases, whether by measuring the dimension of invasive component quantifying its extent or sub-stage according to relationship to muscularis mucosae/vascular plexus [7,8]. Problems rises also considering the trouble in muscularis mucosae detection in the lamina propria: muscolaris mucosae is an erratic structure, hard to identify on biopsy sample, scattered and discontinuous within the tissue with a documented significant topographical variation in different region of the bladder [9-11]. With current procedure there is no identification of the muscolaris mucosae in biopsy sample in up to $35 \%$ of the cases [12].

In this scenario the introduction in routine TURB of novel procedures, such as En-bloc TURB with less artifact and carrying much clear histological information could be helpful in improving our current predictive tool in $\mathrm{T} 1$ management: being able to analyze perfectly oriented and intact surgical specimen is one of the possible way to improve any pathological based stratification, allowing a clear identification of high vs. low risk patients and reducing doubt in T1 management. This novel procedure is also safer in term of recurrence: the En-bloc technique provides normal tissue margins around tumor resection border, reducing risk of incomplete resection or at least making easily to identify excision margin, an assessment that was not practicable in conventional piecemeal based TURB $[13,14]$.

The correlation of invasion depth with tumor aggressiveness has been widely experienced (i.e. melanoma Breslow measurement, Depth on invasion DOI in head and neck squamous cell carcinoma, submucosal invasion depth SID in colorectal carcinoma) and presumably the same approach is applicable to high-grade urothelial bladder carcinoma with results similar to that obtained for other malignancies. In this prospective study we aim to improve our outcome prediction tools, testing an old approach to risk stratification on high quality specimen. The main purpose is to find out if the thickness of invasion is related to tumor recurrence and progression. The utility of en-bloc excisional specimen relies on the possibility of tumor orientation, with clear identification of surgical margin on gross examination: this would allow histological correlation between tumors using histologic landmark, since all anatomic structure are easily identifiable on H\&E slices from oriented sample.

\section{Materials and Methods}

After an internal review board approval, a consecutive series of patients with bladder neoplasms diagnosed on cystoscopy were prospectively enrolled. All patients signed a dedicated informed consent and the study was conducted in accordance with the principles of the declaration of Helsinki. All patients underwent EB-TURB and only patients with pT1 HG disease are included in our study. A second TURB is performed after 4-6 weeks from the first one. All patients receive detailed clinical history and physical examination. Variables recorded include: sex, age, previous history of bladder cancer, number, location and dimensions of lesions during flexible cystoscopy.

\section{Operation Technique}

Under regional anaesthesia, patients are placed in a lithotomy position. Routine cystoscopy is performed to confirm the location, number, and volume of the bladder tumour. Physiologic sodium solution is used for irrigation, the en-bloc resection was performed using Collins knife. Intact tumour is flashed out or extracted with a grasper. Thereafter, adequate coagulation is performed at the tumour ground and surrounding mucosa. Catheter is then inserted after verifying no haemorrhage.

\section{Histological Examination}

Endoscopic specimen, formalin fixed in $10 \%$ buffered formalin solution and unmanipulated for optimum anatomic orientation is sent to pathology lab. Adequate dissection of the gross specimen is essential for accurate measurements: the resected neoplasm is transverse sectioned and whole included in paraffin block and evaluated on ematossiline-eosine section. All slides are digitally acquired using Leica Aperio Scan scope and measured via Aperio Image scope digital slide viewer. Tumor depth is accurately 
evaluated by examining the deepest infiltration measuring the starting from the membrane basalis at a right angle, down to the depth to which tumor cells have invaded submucosa (in $\mathrm{mm}$ ),

deepest point of invasion (Figure 1).

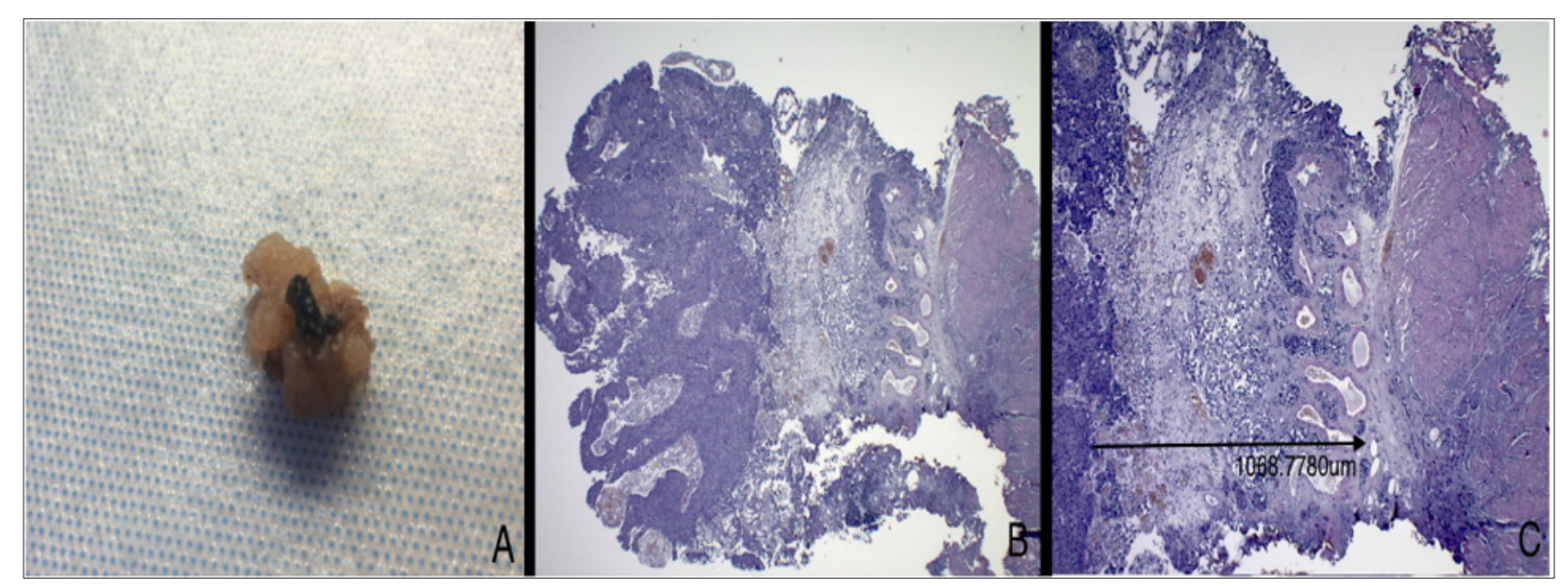

Figure 1: In A. Gross evaluation of a resected specimen, in this case a polypoid lesion, with easily identifying stalk; the deep surgical line of excision is stained with India Ink for margin status assessment purpose. B. Ematossiline-eosine section at 2,5x magnification, showing a perfectly oriented specimen, with loyal representation and clear low-power identification of lamina propria structures. C. Same slides, $4 x$ magnification, the arrow indicate the measurement of depth of invasion identified as the deepest tumor cell in a perpendicular line to the membrane basalis.

\section{Results}

Overall 36 patients with pT1HG disease were enrolled. Out of them EB-resection was adequately performed in $27 / 36(75 \%)$ of the patients. Median age was $72(63 / 78)$ and overall 23/27 (85\%) were males and $5 / 27$ (15\%) were females. Data are shown in Table 1. On cystoscopy $16 / 27$ (59\%) of the patients presented single tumors while 11/27 (41\%) patients presented multifocal lesion. Overall 43 lesions were identified with a median tumor size of $2 . \mathrm{cm}$ $(1 / 4)$ and out of them 36/43 were adequately performed en-bloc. Reasons for not performing the en-bloc technique in the s7 lesions were: $5 / 7$ were electro-fulgurated because to small, $2 / 7$ were neat the ostium.

Table 1: Characteristics of the cohort.

\begin{tabular}{|c|c|}
\hline \multicolumn{2}{|c|}{ Characteristics of the cohort } \\
\hline Patients & 27 \\
\hline Age (years) & $72(63 / 78)$ \\
\hline M/F & $23 / 5$ \\
\hline Cistoscopy diameter (cm) & $2(1 / 4)$ \\
\hline Lesions & \\
\hline Single & $16 / 27$ \\
\hline Multiple & $11 / 27$ \\
\hline Depth of invasion on iTURB $(\mathrm{mm})$ & $1.21(0.45 / 3.24)$ \\
\hline CIS & $6 / 27$ \\
\hline Positive reTURB: & $8 / 27(29 \%)$ \\
\hline LG & $7 / 8$ \\
\hline HG & $1 / 8$ \\
\hline Lesions on reTURB $(\mathrm{mm})$ & 10 \\
\hline Depth of invasion on reTURB $(\mathrm{mm})$ & $0.61(0.21 / 2.2)$ \\
\hline
\end{tabular}

Depth of invasion measurement was feasible in $100 \%$ of the lesions removed with EB-TURB technique. Median depth of invasion was $1.21 \mathrm{~mm}(0.45 / 3.24)$. Overall 6/27 (22\%) presented CIS on histological examination. On re-TURB 8/27(29\%) patients presented residual disease, $7 / 8$ patients presented $\mathrm{pT} 1 \mathrm{HG}$ disease and $1 / 8$ patients presented pTaLG disease on re-TURB. In the 8 patients a total of 10 lesions were resected en-bloc. In $100 \%$ of these patients it was possible to measure depth of invasion and the median value was $0.61 \mathrm{~mm}(0.21 / 2.2)$.

\section{Discussion}

According to actual WHO/ISUP 2016 classification, the group of non-invasive carcinoma comprises tumor with similar biological property as invasive urothelial carcinoma, underscoring the high risk of progression of these pathological entity, ranging from 21 to $50 \%$ [8]. Considering the high variability in clinical outcome within this group of tumor and the lack of well-established risk assessment criteria for recurrence and progression, treatment of pT1 high-grade bladder carcinoma is still challenging, making urgent the needing of individuating outcome predictive elements leading to standardization of management. Along with classical clinical parameter (multiplicity, tumor size, T category, tumor grade, prior recurrence rate and presence of concurrent CIS) histological substaging system has been widely explored and recently depth of invasion has been advocated as the most important prognostic factor in term of disease progression and cancer specific survival [15]. Over the past 20 year subclassification of pT1 stages according to muscularis mucosae invasion seemed to be the most reasonable method for stratification purpose; recently also dimensional approaches proved to be a powerful predictor of tumoral behavior, but to date no definite correlation between neoplastic invasion and time to recurrence and progression has been clearly identified. 
In 1990's Younes was the first to introduce the needing of sub-staging system with prognostic influence in T1 bladder cancer individuating three categories: tumoral invasion located above (a), into (b) and beyond (c) the muscularis mucosae [6]. His group found a significant role of lamina propria invasion in outcome prediction of $\mathrm{T} 1$ tumors with a $75 \% 5$ year survival for $\mathrm{T} 1 \mathrm{a}$ and $\mathrm{T} 1 \mathrm{~b}$ and an $11 \%$ for $\mathrm{T} 1 \mathrm{c}$ opening the way to many other different systems in order to get the best fitting subdivision of this heterogeneous group of tumor. Other anatomy-based systems sub-classify NMIBC according to presence of cancer invasion near muscularis mucosae (pT1b) or absence (pT1a) [16] or more simply in above or into (pT1a) and beyond (pT1b) [17]. Lawless et al. focusing on papillary exophytic subtype with its peculiar stalk architecture, proposes to sub-stage by separating invasion of the lamina propria into the stalk from invasion of the base, either focal or extensive (basing on a $1 \mathrm{~mm}$ threshold), with significant results in term of progression [18]. Along these lines other used a sizebased approach: Chang measurement focus on maximum extent of invasion [19], while other method recognizes microinvasive (pT1-m) versus extensive (pT1-e) invasive bladder tumor in 1 highpower field with a $0,5 \mathrm{~mm}$ threshold [20]. Recently the $1 \mathrm{~mm}$ cut-off in 20x HPF multidirectional measurement adopted in the ROL (Rete Oncologica Lombarda) system [21] proved to be feasible and highly reproducible.

However, the lack of uniformity between these methods make data comparison difficult and to date no reliable correlation in term of disease recurrence and progression has been found. Must be considered that pathological staging of urothelial bladder carcinoma on specimens obtained with traditional TURB is often inaccurate due to fragmented tumor samples, with poor anatomic orientation, severe thermal artifact and crush effects related to the surgical procedure. These technique intrinsic artifacts are most likely the main reason why all measurement based sub staging system failed [22,23] and lead also to high inter-observer variability with subsequent risk of understaging and misleading therapeutic decisions. Since its introduction by Edwin Beer [24] endoscopic management of bladder tumor remains the Gold standard, and to date, TURB techniques allow not only diagnosis but, in many cases, definitive treatment by mean of total removal of bladder cancer. According to Herr quality assessment of a good TURB must evaluate the complete resection, presence of deep muscle in the sample and the rate of recurrence [25]. Despite its long standing application, even in experienced specialist, TURB resection is sometimes inadequate: consider the case of multiple tumor, tumors with large bulk, or patients features such as habitus or aesthetic risk, or even procedure inherent complication such as bleeding or bladder perforation [26,27].

In surgical practice despite the great variation in techniques and quality of TURB no consensus has been found in adequacy of Turb specimen sampling [28]. Both CUA (Canadian Urological Association) and NICE (National Institute for Health and Care Excellence) guidelines emphasize the requirement of obtaining muscle within the specimen. NICE also recommends repeating the procedure within 6 weeks if histologically none is identified [29]. Although the complete one time removal of all bladder cancer by
TURB is the goal, this is not always easy to reach. A second TURB is recommended for the following cases: incomplete initial TURB, no muscle in the specimen after initial resection (with exception of Ta low grade tumors), in case of T1 tumor and all high grade tumors [30]. Increasing evidence supports EB-TURB improvement of specimen quality; the presence of muscular propria in neoplastic resection is crucial for correct diagnosis and in one study detrusor muscle was clearly identified in $94.4 \%$ initial specimen from En-Bloc TURBT, whereas only in 60\% from conventional TURB biopsy [14]. In a pathological point of view En Bloc TURB can both improve the surgical management, reducing the needing of a second look TURB, and facilitate the sample evaluation, especially for what concern depth of tumor invasion and margins status, allowing accurate histological based risk stratification. In the present study we proved the feasibility of neoplastic depth measurement when performing EB-TURB. In this pilot study all the resected lesions it was passible of depth of invasion evaluation. Moreover, the measurement was simple and straightforward. However, it is important to underline that in $25 \%$ of the patients it was not possible to perform an EBTURB and were excluded from the study because of large tumour $(>5 \mathrm{~cm})$ and fragmentation of the tissue when removed from the bladder. The present study is the only available evidence to our knowledge describing the measurement in pT1 high grade bladder cancer of invasion deepness on EB-TURB specimens.

\section{Conclusion}

Despite the paucity of high-level evidence regarding correlation of neoplastic invasion with recurrence and progression in pT1NMIBC, histological sub-staging is still attractive and the latest AJCC Cancer staging manual strongly recommend reporting further information in pT1 diagnosis record, encouraging anatomybased subclassification and tumoral invasion measurements. We are deeply confident that the introduction in common surgical practice of much representative tumor sample, obtained with tissue respectful techniques such as en-bloc TURB, could improve our outcome prediction tools: the possibility to analyze perfectly oriented specimen, with easily recognizable lamina propria structure and no doubt in invasion assessment trough reproducible measurement method, is the only way to compare tumor, and validate or disprove the role of neoplastic invasion thickness in $\mathrm{mm}$.

In line with other epithelial malignancies, staging according to depth of invasion hold the promise to improve our understanding in this heterogeneous group of neoplasm. Target of this prospective study is to get over problems affecting the TURB procedure in order to verify if tumoral thickness is actually an outcome predictive variable, and if so, to set a significant cut-off. The precise topographical localization of neoplastic cell and the clear identification of tumoral relationship with submucosal structures would surely reduce interobserver variability in pT1 diagnosis, with no doubt in invasion assessment and measurement, allowing reliable stratification of recurrence and progression and thus providing basis for rational therapeutic approach to high-grade non muscle invasive carcinoma behavior. Comparison of measurements will eventually reveal statistical correlation between the depth of invasion measurement and other validated clinical parameters or 
assess a role as independent prognostic factor for the histological parameter.

To appreciate the potential benefit of this approach it would be necessary to enroll a large number of patients. At the time of submission due to the limited follow-up no correlation is allowed yet, but histological information obtained from en-bloc TURB are so thick that we are truly confident will confirm our expectation.

\section{Acknowledgement}

This research did not receive any specific grant from funding agencies in the public, commercial, or not-for profit sectors.

\section{References}

1. Siegel RL, Miller KD, Jemal A (2015) Cancer statistics, 2015. CA Cancer J Clin 65(1): 5-29.

2. Wassim Kassouf, Samer L Traboulsi, Girish S Kulkarni, Rodney H Breau, Alexandre Zlotta, et al. (2015) CUA guidelines on the management of nonmuscle invasive bladder cancer. Can Urol Assoc J 9(9-10): E690-E704.

3. Cookson MS, Chang SS, Wells N, Parekh DJ, Smith JA (2003) Complications of radical cystectomy for nonmuscle invasive disease: comparison with muscle invasive disease. J Urol 169(1): 101-104.

4. Sylvester RJ, van der Meijden AP, Oosterlinck W, Witjes JA, Bouffioux C, et al. (2006) Predicting recurrence and progression in individual patients with stage Ta T1 bladder cancer using EORTC risk tables: A combined analysis of 2596 patients from seven EORTC trials. Eur Urol 49(3): 466500.

5. Xylinas E, Kent M, Kluth L, Pycha A, Comploj E, et al. (2013) Accuracy of the EORTC risk tables and of the CUETO scoring model to predict outcomes in non-muscle-invasive urothelial carcinoma of the bladder. Br J Cancer 109(6): 1460-1466.

6. Younes M, Sussman J, True LD (1990) True, The usefulness of the level of the muscularis mucosae in the staging of invasive transitional cell carcinoma of the urinary bladder. Cancer 66(3): 543-548.

7. Amin MB, Greene FL, Edge SB, Compton CC, Gershenwald JE, et al. (2017) The eighth edition AJCC cancer staging manual: Continuing to build a bridge from a population-based to a more "personalized" approach to cancer staging. CA Cancer J Clin 67(2): 93-99.

8. Moch H, Cubilla AL, Humphrey PA, Reuter VE, Ulbright TM (2016) The 2016 WHO classification of tumours of the urinary system and male genital organs-Part A: Renal, penile and testicular tumours. Eur Urol 70(1): 93-105.

9. Dixon JS, Gosling JA (1983) Histology and fine structure of the muscularis mucosae of the human urinary bladder. J Anat 136(Pt 2): 265-271.

10. Engel P, Anagnostaki L, Braendstrup $O$ (1992) The muscularis mucosae of the human urinary bladder. Implications for tumor staging on biopsies. Scand J Urol Nephrol 26(3): 249-252.

11. Paner GP, Ro JY, Wojcik EM, Venkataraman G, Datta MW, et al. (2007) Further characterization of the muscle layers and lamina propria of the urinary bladder by systematic histologic mapping: Implications for pathologic staging of invasive urothelial carcinoma. Am J Surg Pathol 31(9): 1420-1429.

12. Orsola A, Trias I, Raventós CX, Español I, Cecchini L, et al. (2005) Initial high-grade T1 urothelial cell carcinoma: Feasibility and prognostic significance of lamina propria invasion microstaging (T1a/b/c) in BCGtreated and BCG-non-treated patients. Eur Urol 48(2): 231-238.
13. Ray ER, O'Brien TS (2007) Should urologists be spending more time on the golf course? BJU Int 100(4): 728-729.

14. Upadhyay R, Kapoor R, Srivastava A, Krishnani N, Mandhani A (2012) Does En-bloc transurethral resection of bladder tumor give a better yield in terms of presence of detrusor muscle in the biopsy specimen? Indian J Urol 28(3): 275-279.

15. Martin-Doyle W, Leow JJ, Orsola A, Chang SL, Bellmunt J (2015) Improving selection criteria for early cystectomy in high-grade 1 bladder cancer: A meta-analysis of 15,215 patients. J Clin Oncol 33(6): 643-650.

16. Hasui Y, Osada Y, Kitada S, Nishi S (1994) Significance of invasion to the muscularis mucosae on the progression of superficial bladder cancer. Urology 43(6): 782-786.

17. Holmäng S, Hedelin H, Anderström C, Holmberg E, Johansson SL (1997) The importance of the depth of invasion in stage T1 bladder carcinoma: A prospective cohort study. J Urol 157(3): 800-803.

18. Lawless M, Gulati R, Tretiakova M (2017) Stalk versus base invasion in pT1 papillary cancers of the bladder: Improved substaging system predicting the risk of progression. Histopathology 71(3): 406-414.

19. Chang WC, Chang YH, Pan CC (2012) Prognostic significance in substaging of T1 urinary bladder urothelial carcinoma on transurethral resection. Am J Surg Pathol 36(3): 454-461.

20. van Rhijn BW, van der Kwast TH, Alkhateeb SS, Fleshner NE, van Leenders GJ, et al. (2012) A new and highly prognostic system to discern T1 bladder cancer substage. Eur Urol 61(2): 378-384.

21. Carlo Patriarca, Rodolfo Hurle, Marco Moschini, Massimo Freschi, Piergiuseppe Colombo, et al. (2016) Usefulness of pT1 substaging in papillary urothelial bladder carcinoma. Diagn Pathol 11: 6.

22. Hu Z, Mudaliar K, Quek ML, Paner GP, Barkan GA (2014) Measuring the dimension of invasive component in pT1 urothelial carcinoma in transurethral resection specimens can predict time to recurrence. Ann Diagn Pathol 18(2): 49-52.

23. Hansel DE, Amin MB, Comperat E, Cote RJ, Knüchel R, et al. (2013) A contemporary update on pathology standards for bladder cancer: Transurethral resection and radical cystectomy specimens. Eur Urol 63(2): 321-332.

24. Herr HW (2005) Legacy of edwin beer: Fulguration of papillary bladder tumors. J Urol 173(4): 1087-1089.

25. Herr HW, Donat SM (2008) Quality control in transurethral resection of bladder tumours. BJU Int 102(9 Pt B): 1242-1246.

26. Traxer O, Pasqui F, Gattegno B, Pearle MS (2004) Technique and complications of transurethral surgery for bladder tumours. BJU Int 94(4): 492-496.

27. Dick A, Barnes R, Hadley H, Bergman RT, Ninan CA (1980) Complications of transurethral resection of bladder tumors: prevention, recognition and treatment. J Urol 124(6): 810-811.

28. Richards KA, Smith ND, Steinberg GD (2014) The importance of transurethral resection of bladder tumor in the management of nonmuscle invasive bladder cancer: a systematic review of novel technologies. J Urol 191(6): 1655-1664.

29. Nicholas E Power, Jonathan Izawa (2016) Comparison of guidelines on non-muscle invasive bladder cancer (EAU, CUA, AUA, NCCN, NICE). Bladder Cancer 2(1): 27-36.

30. Babjuk M, Burger M, Zigeuner R, Shariat SF, van Rhijn BW, et al. (2013) EAU guidelines on non-muscle-invasive urothelial carcinoma of the bladder: update 2013. Eur Urol 64(4): 639-653. 
ISSN: 2574-1241

DOI: $10.26717 / B J S T R .2019 .13 .002372$

Roberto Giulianelli, Ludovica De V. Biomed J Sci \& Tech Res

CC (i) This work is licensed under Creative

Submission Link: https://biomedres.us/submit-manuscript.php

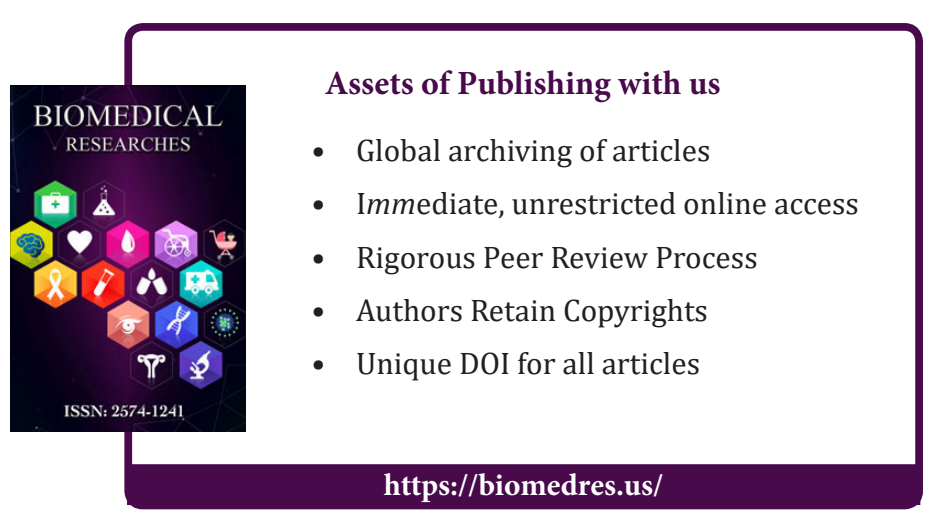

